\title{
Uterine Smooth Muscle Tumor of Uncertain Malignant Potential: Clinicopathologic-Sonographic Characteristics, Follow-Up and Recurrence
}

\author{
Besim Haluk Bacanakgil ${ }^{\mathrm{a}}$, Mustafa Deveci ${ }^{\mathrm{a}, \mathrm{b}}$, Emine Karabuk ${ }^{\mathrm{a}}$, \\ Zeynep Soyman ${ }^{\mathrm{a}}$
}

\begin{abstract}
Background: Uterine smooth muscle tumor of uncertain malignant potential (STUMP) is rare tumor, and regarded as sub-classification in uterine smooth muscle tumors between benign and malignant criteria. In this study, we evaluated characteristics of cases with STUMP diagnosis in a 10 -year period.
\end{abstract}

Methods: We retrospectively evaluated medical records of patients with histopathological STUMP diagnosis in Istanbul Training and Research Hospital, a tertiary center. We analyzed preoperative demographic, clinical features and postoperative follow-up. Preoperative sonographic data were re-evaluated.

Results: The mean age was 42 years. One patient was postmenopausal, and five patients were premenopausal. All of them had a complaint of meno-metrorrhagia. We re-evaluated preoperative sonographic images of patients, and defined $83.3 \%$ as well-defined margins, $66.7 \%$ hyperechoic, $100 \%$ heterogeneous, $66.7 \%$ noncystic, $50 \%$ calcification and $66.7 \%$ acoustic shadowing. Pathologic features showed mean number of mitosis 8 , mild atypia $66.7 \%$, and necrosis $33.3 \%$. In a 24-year-old unmarried female patient with myomectomy, we detected recurrance of tumor in sonographic and MRI studies after 11 months, and confirmed the diagnosis via trucut biopsy. There was no relevance between sonographic findings and atypia, necrosis and mitosis. The recurrence was not in relationship with mitosis, degree of atypia and necrosis. We found no relevance between tumor diameter and mitosis, atypia, necrosis and recurrence.

Conclusions: STUMP is classified as an intermediate form, histopathologically so calling it benign or malignant for sure is not possible. Singulary, solidity, hyperechogenicity, heterogenecity and features of acoustic shadowing and margins can guide us to preoperative sonographic diagnosis. Recurrence/metastasis after many years from

Manuscript accepted for publication May 10, 2017

${ }^{\mathrm{a} G y n e c o l o g y}$ and Obstetrics Clinic, Istanbul Training and Research Hospital, Istanbul, Turkey

${ }^{b}$ Corresponding Author: Mustafa Deveci, Gynecology and Obstetrics Clinic, Istanbul Training and Research Hospital, Istanbul, Turkey.

Email: mustafa.deveci@gmail.com

doi: https://doi.org/10.14740/wjon1031w operation can be seen, and those patients should be followed long term.

Keywords: Uterine smooth muscle tumor; STUMP; Sonography

\section{Introduction}

Uterine smooth muscle tumor of uncertain malignant potential (STUMP) is a rare tumor.

Uterine smooth muscle tumors are classified as benign leiomyoma and malignant leiomyosarcoma regarding presence of tumor cell necrosis, cytological atypia and mitotic activity. STUMP is regarded in World Health Organization (WHO) classification as smooth muscle tumors between benign and malignant criteria [1].

STUMP is seen mostly in age of mid-forties, who has been operated with preoperative leiomyoma diagnosis. Risk factors and prognosis are not fully understood, but in longterm follow-up, there is a potential of recurrence or metastasis.

In this study, we evaluated prognosis, sonographic and clinicopathologic features of cases with histopathological STUMP diagnosis.

\section{Materials and Methods}

We retrospectively evaluated medical records of patients with histopathological STUMP diagnosis between 2005 and 2015 in Istanbul Training and Research Hospital, a tertiary center. We analyzed preoperative demographic, clinical and histopathological features, and postoperative follow-up. Preoperative sonographic data were re-evaluated.

SPSS 15.0 for Windows was used for statistical analysis. Descriptive statistics were identified as number and percentage for categorical variables, and mean, standard deviation, minimum and maximum for numeric variable. Comparison of two independent groups was made with Mann-Whitney U test. The ratio in two groups for categorical variables was analyzed with Fisher's exact test. Numerical variables were analyzed by Spearman correlation analysis due to failure to fulfill the parametric test condition. Statistical significance level has been ac- 
Table 1. Case-Based Features

\begin{tabular}{|c|c|c|c|c|c|c|}
\hline & Case 1 & Case 2 & Case 3 & Case 4 & Case 5 & Case 6 \\
\hline Age & 48 & 44 & 40 & 43 & 52 & 24 \\
\hline Gravida & 12 & 8 & 2 & 4 & 7 & 0 \\
\hline Menopausal state & Post & Pre & Pre & Pre & Pre & Pre \\
\hline Complaint & MM & MM & $\mathrm{MM}$ & MM & MM & MM and pelvic pain \\
\hline Number of tumor/myoma & 5 & 1 & 1 & 1 & 1 & 2 \\
\hline Margins of the tumor & Ill-defined & Well-defined & Well-defined & Well-defined & Well-defined & Well-defined \\
\hline Echogenicity & Mixed & Mixed & Hyperechoic & Hyperechoic & Hyperechoic & Hyperechoic \\
\hline Appearance & Heterogenous & Heterogenous & Heterogenous & Heterogenous & Heterogenous & Heterogenous \\
\hline Cystic form & + & + & - & - & - & - \\
\hline \multicolumn{7}{|l|}{ Pathology } \\
\hline Procedure & $\mathrm{TAH}+\mathrm{BSO}$ & $\mathrm{TAH}+\mathrm{BSO}$ & Myomectomy & $\mathrm{TAH}+\mathrm{BSO}$ & $\mathrm{TAH}+\mathrm{BSO}$ & Myomectomy \\
\hline Tumor diameter $(\mathrm{cm})$ & 3.5 & 8 & 7 & 20 & 10 & 8 \\
\hline Localization & Intramural & Submucous & Intramural & Intramural & Intramural & Intramural \\
\hline Number of mitosis (n/10 HPF) & 4 & 17 & 8 & 5 & 8 & 6 \\
\hline Atypia & Mild & Moderate & Moderate & Moderate & Mild & Moderate \\
\hline Necrosis & + & + & - & - & - & - \\
\hline Follow-up (months) & 120 & 119 & NR & 38 & 29 & 11 \\
\hline
\end{tabular}

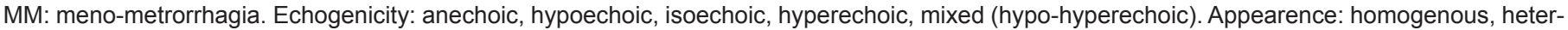
ogenous. +: present, -: absent. NR: not to be reached.

cepted as $\mathrm{P}<0.05$.

\section{Results}

Case-based features and their statistical analysis of six patients with STUMP diagnosis are presented in Tables 1 and 2. The mean age was 42 years. One patient was postmenopausal, and five patients were premenopausal. All of them had a complaint of meno-metrorrhagia. Mean gravida was 5.5, and mean parity was 3.5. We re-evaluated preoperative sonographic tumor images, and defined $83.3 \%$ as well-defined tumor capsule, $66.7 \%$ hyperechoic, $100 \%$ heterogeneous, $66.7 \%$ non-cystic, $50 \%$ calcification and $66.7 \%$ acoustic shadowing. We performed total abdominal hysterectomy (TAH) and bilateral salpingooophorectomy (BSO) in $66.7 \%$ and myomectomy in $33.3 \%$ of the patients. In the histopathology, mean tumor diameter was $9.4 \mathrm{~cm}$, localization was intramural in $83.3 \%$, mean number of mitosis was 8 (in 10 high power fields (HPF)), mild atypia in $66.7 \%$ and necrosis in $33.3 \%$. Mean follow-up time was 63.4 months. We could not reach one patient postoperatively. In a 24-year-old nulliparous woman who had undergone myomec- tomy, we detected recurrance of tumor in sonography and MRI studies after 11-month follow-up, and confirmed the diagnosis via tru-cut biopsy.

There was no statistically significant relevance between sonographic findings and atypia, necrosis and mitosis number $(\mathrm{P}>0.05)$. The recurrence was not in relationship with mitosis, degree of atypia and necrosis. We found no relevance between tumor diameter and mitosis, atypia, necrosis and recurrence. In the developing recurrence case, there were two tumors with largest diameter of $8 \mathrm{~cm}$, moderate atypia, 6 mitosis, and no necrosis. Another myomectomy case was similar: one tumor with largest diameter of $7 \mathrm{~cm}$, moderate atypia, 8 mitosis, and no necrosis.

\section{Discussion}

STUMP, which is considered a subtype of uterine smooth muscle tumors, is a rare tumor, so the incidance is not well known. Also there is no consensus on histopathological description.

Clinical signs and symptoms are similar with leiomyoma and leiomyosarcoma [2-4]. Those are pelvic pain, anormal 
Table 2. Statistical Analysis of Case-Based Features

\begin{tabular}{|c|c|}
\hline Age, mean \pm SD (min. - max.) & $41.8 \pm 9.7(24-52)$ \\
\hline Gravida, mean \pm SD (min. - max.) & $5.5 \pm 4.4(0-12)$ \\
\hline Parity, mean \pm SD (min. - max.) & $3.5 \pm 2.2(0-6)$ \\
\hline \multicolumn{2}{|l|}{ Menapausal state, $\mathrm{n}(\%)$} \\
\hline Postmenapause & $1(16.7)$ \\
\hline Premenapause & $5(83.3)$ \\
\hline \multicolumn{2}{|l|}{ Complaint, n (\%) } \\
\hline Meno-metrorrhagia & $6(100)$ \\
\hline \multicolumn{2}{|l|}{ Sonography } \\
\hline Number of tumor/myoma, mean $\pm \mathrm{SD}$ (min. - max.) & $1.8 \pm 1.6(1-5)$ \\
\hline \multicolumn{2}{|l|}{ Margins of the tumor, $\mathrm{n}(\%)$} \\
\hline Ill-defined & $1(16.7)$ \\
\hline Well-defined & $5(83.3)$ \\
\hline \multicolumn{2}{|l|}{ Tumor echogenicity, $\mathrm{n}(\%)$} \\
\hline Hyperechoic & $4(66.7)$ \\
\hline Mixed & $2(33.3)$ \\
\hline \multicolumn{2}{|l|}{ Tumor heterogenicity, $\mathrm{n}(\%)$} \\
\hline Heterogenous & $6(100)$ \\
\hline \multicolumn{2}{|l|}{ Cystic appearance, $\mathrm{n}(\%)$} \\
\hline+ & $2(33.3)$ \\
\hline- & $4(66.7)$ \\
\hline \multicolumn{2}{|l|}{ Calcification n $(\%)$} \\
\hline+ & $3(50.0)$ \\
\hline- & $3(50.0)$ \\
\hline \multicolumn{2}{|l|}{ Acoustic shadowing, $\mathrm{n}(\%)$} \\
\hline+ & $4(66.7)$ \\
\hline- & $2(33.3)$ \\
\hline Free fluid, n (\%) & 0 \\
\hline \multicolumn{2}{|l|}{ Pathology } \\
\hline \multicolumn{2}{|l|}{ Procedure, n (\%) } \\
\hline Myomectomy & $2(33.3)$ \\
\hline $\mathrm{TAH}+\mathrm{BSO}$ & $4(66.7)$ \\
\hline Tumor diameter $(\mathrm{cm})$, mean $\pm \mathrm{SD}$ (min. - max.) & $9.4 \pm 5.6(3.5-20)$ \\
\hline \multicolumn{2}{|l|}{ Localization, $\mathrm{n}(\%)$} \\
\hline Intramural & $5(83.3)$ \\
\hline Submucous & $1(16.7)$ \\
\hline Mitosis count (n/10 HPF), mean $\pm \mathrm{SD}$ (min. - max.) & $8.0 \pm 4.7(4-17)$ \\
\hline \multicolumn{2}{|l|}{ Atypia, n (\%) } \\
\hline Mild & $2(33.3)$ \\
\hline Moderate & $4(66.7)$ \\
\hline \multicolumn{2}{|l|}{ Necrosis, $\mathrm{n}(\%)$} \\
\hline+ & $2(33.3)$ \\
\hline- & $4(66.7)$ \\
\hline \multicolumn{2}{|l|}{ Final situation $\mathrm{n}(\%)$} \\
\hline Recurrence & $1(20)$ \\
\hline Disease-free & $4(80)$ \\
\hline NR & 1 \\
\hline Follow-up (months), mean \pm SD (min. - max.) & $63.4 \pm 52.1(11-120)$ \\
\hline
\end{tabular}


uterine bleeding, pelvic mass, and pelvic pressure sensation or combination of them. Mean age is 45 years [2-4]. Most of the patients are premenopausal [4]. In our cases, mean age was 42. Of the patients, $83.3 \%$ were premenapausal. All of them had meno-metrorrhagia.

Preoperative STUMP diagnosis or differentiation from leiomyoma-leiomyosarcoma with imaging modalities is not easy. Bonneau et al published the first study about this in 2013 [5]. In this study, they have compared sonography and MRI findings of 85 leiomyoma and 23 malignant mesenchymal tumor (MMT) and STUMP cases, and reported that presence of single tumor, absence of acoustic shadowing and presence of free fluid are associated with MMT/STUMP. We also retrospectively evaluted our sonography images according to their criteria: four of cases had single tumor, there were no acoustic shadowing in two cases, and none of cases had free fluid. Our sonographic findings showed $83.3 \%$ well-defined, $66.7 \%$ hyperechoic, $100 \%$ heterogenous, $66.7 \%$ noncystic, $50 \%$ calcification and $66.7 \%$ acoustic shadowing.

Uterine smooth muscle tumors have historically been distinct in benign leiomyomas and malignant leiomyosarcomas on the basis of cytological atypia, mitotic rate and presence or absence of tumor cell necrosis (CTCN) [6]. The Stanford criteria for the histologic diagnosis of leiomyosarcoma reported by Bell et al include at least two of the following criteria: diffuse moderate-to-severe atypia, a mitotic count of at least 10 mitotic figures/10 HPF and tumor cell necrosis criteria; absence of necrosis and atypia, $\leq 4$ mitosis indicating benign leiomyoma. There is no consensus on diagnostic criteria of STUMP in literature. WHO classification indicates that a uterine SMT not diagnosed unequivocally as benign or malignant should be defined as STUMP. The STUMP tumors were subdivided by Bell et al [6] into three groups: 1) atypical leiomyoma with low risk of recurrence - diffuse moderate-tosevere atypia, < 10 mitosis/10 HPF and no CTCN; 2) atypical leiomyoma but limited experience-severe atypia, $<20$ mitosis/10 HPF, no CTCN; and 3) smooth muscle tumor of low malignant potential has CTCN, mitosis less than 10/10 HPF, absent to mild nuclear atypia. Guntupalli et al [2] had diagnosis of STUMP in one of the following criteria: tumor necrosis $(+)$, no atypia, mitosis $\leq 10 / 10 \mathrm{HPF}$; diffuse atypia $(+)$, no tumor necrosis, mitosis $\leq 10 / 10 \mathrm{HPF}$; no tumor necrosis, no atypia, mitosis $\geq 20 / 10 \mathrm{HPF}$; cellularity or hypercellularity with mitosis $\geq 4 / 10 \mathrm{HPF}$; irregular margins or vascular invasion in peripheral side of tumor.

D'Angelo and Prat [7] described the following criteria for STUMP: tumor necrosis in typical leiomyoa; necrosis and $\geq$ 10 mitosis/10 HPF or remarkable diffuse atypia; remarkable diffuse or focal atypia and borderline mitosis; and hardly classified necrosis. Generally, STUMP diagnosis is decided after myomectomy or hysterectomy according to three histological criteria of the Stanford. If the tumor does not meet criteria for leiomyosarcoma, and has combinations of Stanford's criteria, STUMP diagnosis is accurate.

Reported recurrence rate for STUMP is 7-27\% [8, 9]. Tumor necrosis, degree of atypia and mitosis are correlated with malignant behavior, but relationship with recurrence is not clear. Because, the recurrence is observed in single or variable combinations and degrees of those [2-4, 8-12]. Therefore, it is not so easy to foresee biological behavior, metastatic potential or prognosis of STUMP. Recurrence tumor is either STUMP or leiomyosarcoma, and can occur in uterus, pelvis, retroperitoneum, lung, liver and bone [2, 3, 9-12]. In patients with STUMP, tumor growth is slower, and recurrence is often delayed for a mean of 51 months after the initial diagnosis. Furthermore, the clinical course is often delayed, with a median survival of 61.5 months [8]. Five years survival is $92-100 \%$ [4, 12]. In our study, mean follow-up duration was 63 months, and recurrence rate was $20 \%$. Five patients were still alive, and four of them were disease-free. In our recurrence case, number of mitosis was 6 , with moderate atypia and no necrosis. In this case, recurrence has been diagnosed after 11 months as STUMP.

The recurrence ratio after hysterectomy and myomectomy is similar [2]. Recurrence rate after myomectomy is 6.6\% [8]. In post-myomectomy STUMP diagnosis can be performed second myomectomy or hysterectomy according to patients condition. The patient's age, fertility, pathological properties of tumor, number of recurrence, size and location of the tumor should be carefully considered before deciding the procedure. Successful pregnancy rate after myomectomy is $52 \%$ [8]. Patients who desire pregnancy should be informed about potential morbidity and mortality of tumor. Treatment options for recurrence in addition to surgery include adjuvant chemotherapy, radiotherapy, and progestins $\mathrm{GnRH}$ analogs [2-4, 8-11]. Our recurrence case was unmarried, 24 years old, and had multiple nodules with the biggest one being $12 \mathrm{~cm}$, and we recommended surgery and adjuvant therapy after detailed briefing.

The patients should undergo baseline assessment and regular follow-up every 6 months for the first 5 years, then annual surveillance for the next 5 years [8]. Follow-up visits should consist of a history, general and pelvic examination, and imaging studies annually including chest radiography, pelvic ultrasound, MRI and/or PET-CT, to detect recurrences.

\section{Conclusion}

STUMP is a rare heterogenous tumor. All of the cases were operated with benign preoperative gynecological diagnosis and final diagnosis has been made histopathologically. It is not easy to foresee the biological behavior pattern and prognosis of STUMP. It is classified as an intermediate form histopathologically, so calling it benign or malignant for sure is not possible. We have limited literature information for STUMP. The final histopathological diagnosis is usually made according to Stanford criteria. Preoperatively, sonographic discrimination from leiomyoma or leiomyosarcoma is not possible definitely. Although our case number is limited, singulary, solidity, hyperechogenicity, heterogenocity and features of acoustic shadowing and margins can guide us to preoperative sonographic diagnosis. STUMP is clinically benign, but recurrence/metastasis after many years from operation can be seen, and those patients should be followed long term.

\section{References}

1. Tavassoli FA, Devilee P. World Health Organization Clas- 
sification of Tumours: Tumours of the Breast and Female Genital Organs. Lyon: International Agency for Research on Cancer Press; 2003. pp. 236-239.

2. Guntupalli SR, Ramirez PT, Anderson ML, Milam MR, Bodurka DC, Malpica A. Uterine smooth muscle tumor of uncertain malignant potential: a retrospective analysis. Gynecol Oncol. 2009;113(3):324-326.

3. Ip PP, Cheung AN, Clement PB. Uterine smooth muscle tumors of uncertain malignant potential (STUMP): a clinicopathologic analysis of 16 cases. Am J Surg Pathol. 2009;33(7):992-1005.

4. Ng JS, Han A, Chew SH, Low J. A clinicopathologic study of uterine smooth muscle tumours of uncertain malignant potential (STUMP). Ann Acad Med Singapore. 2010;39(8):625-628.

5. Bonneau C, Thomassin-Naggara I, Dechoux S, Cortez A, Darai E, Rouzier R. Value of ultrasonography and magnetic resonance imaging for the characterization of uterine mesenchymal tumors. Acta Obstet Gynecol Scand. 2014;93(3):261-268.

6. Bell SW, Kempson RL, Hendrickson MR. Problematic uterine smooth muscle neoplasms. A clinicopathologic study of 213 cases. Am J Surg Pathol. 1994;18(6):535558.
7. D'Angelo E, Prat J. Uterine sarcomas: a review. Gynecol Oncol. 2010;116(1):131-139.

8. Vilos GA, Marks J, Ettler HC, Vilos AG, Prefontaine M, Abu-Rafea B. Uterine smooth muscle tumors of uncertain malignant potential: diagnostic challenges and therapeutic dilemmas. Report of 2 cases and review of the literature. J Minim Invasive Gynecol. 2012;19(3):288-295.

9. Dall'Asta A, Gizzo S, Musaro A, Quaranta M, Noventa M, Migliavacca C, Sozzi G, et al. Uterine smooth muscle tumors of uncertain malignant potential (STUMP): pathology, follow-up and recurrence. Int J Clin Exp Pathol. 2014; 7(11):8136-8142.

10. Shapiro A, Ferenczy A, Turcotte R, Bruchim I, Gotlieb WH. Uterine smooth-muscle tumor of uncertain malignant potential metastasizing to the humerus as a highgrade leiomyosarcoma. Gynecol Oncol. 2004;94(3):818820.

11. Esch M, Teschner M, Braesen JH. Pulmonary Metastases of a Uterine Smooth Muscle Tumour with Undefined Malignancy Potential. Geburtshilfe Frauenheilkd. 2014;74(3):288-292.

12. Peters WA, 3rd, Howard DR, Andersen WA, Figge DC. Uterine smooth-muscle tumors of uncertain malignant potential. Obstet Gynecol. 1994;83(6):1015-1020. 